\title{
Optimization and screening of solid lipid nanoparticle production for gene delivery by factorial design and response surface methodology
}

\author{
Hasan Akbaba $\cdot$ Melike Ozder \\ Department of Pharmaceutical Biotechnology, Faculty of Pharmacy, Ege University, İzmir, Turkey
}

\section{ABSTRACT}

Aim: A successful gene therapy requires a delivery system for overcoming various biological barriers. For this, we adapted the factorial design and response surface methodology to the cationic solid lipid nanoparticle production process.

Methods: Screening and optimization of formulations were carried out with factorial design with 3 factors and 3 levels using Box-Behnken Design. Then, solid lipid nanoparticles were physicochemically characterized. Furthermore, optimal SLN formulation is examined in terms of complex formation with plasmid DNA, its protection potential against nucleases, cytotoxicity profile, and storage stability.

Results: Response-surface analyses demonstrated that the selected quadratic model holds significance for particle size and zeta potential. The interaction of independent variables was statistically determined. Optimization and prediction were performed using obtained second-order polynomial equations. Optimal formulation and complexes were found to be nanosized, positively charged and their polydispersity-index values below 0.3 as an indicator of being monodispersed. Cytotoxicity of the optimal formulation is compatible for further studies and no significant increase was observed in particle size until day 21 and until day 60 for polydispersity-index.

Conclusion: Optimal formulation provides a good basis as a gene delivery system was produced with developed systematic. Briefly, this methodology could be used to obtain SLNs with desired conditions.

Keywords: Solid lipid nanoparticle, factorial design, response surface method, gene delivery.

Hasan Akbaba,

Department of Pharmaceutical Biotechnology, Faculty

of Pharmacy, Ege University, İmir, Turkey

E-mail: hasan.akbaba@ege.edu.tr

Received: 2020-08-31 / Revised: 2020-09-23

Accepted: 2020-09-26 / Published online: 2021-01-01

\section{Introduction}

According to the U.S. Food and Drug Administration (FDA), gene therapy purpose to change or control the expression of a gene or to make adjustments to the biological features of living cells for therapeutic use [1].
Successful gene therapy requires a gene delivery system for overcoming various biological barriers and the effects of the environment. The gene delivery system must protect the genetic material from lysosomal degradation when taken into cell cytoplasm with endocytosis and then be able to transcend the nuclear membrane as a biological barrier. Genetic material also should be protected against harsh environmental conditions such as $\mathrm{pH}$ and temperature change, redox reactions [25]. 
Various gene transfer techniques are applied. They are divided into viral and non-viral techniques. As viral techniques; adenoviruses, adeno-associated viruses, lentiviruses, and retroviruses are often used for gene delivery. Non-viral techniques contain; inorganic particles (calcium phosphate, silica, gold, magnetic), synthetic or natural biodegradable particles (polymeric, cationic lipid, peptidebased vectors) and physical methods (needle injection, ballistic DNA injection, electroporation, sonoporation, photoporation, magnetofection, hydroporation) [6-8].

Although viral techniques are mostly used in clinical trials, some of their difficulties caused alternative techniques to be considered. These difficulties are biosafety problems (high immunogenicity and potential insertional mutagenesis) and limited gene package capacity [9-11].

One of the non-viral methods, solid lipid nanoparticles (SLN); are non-toxic carrier systems stabilized by surfactants and made from solid lipids for controlled and targeted delivery. Their advantageous features are, biocompatibility, assembling of hydrophilic and hydrophobic drugs, maintain the release of the drug and avoid photochemical, chemical, or oxidative degradation [12]. These systems have several advantages such as improved transfection, reduced immunogenicity, large gene package size, ease of manufacture, protection from degradation, prolong circulation of the nanoparticles (NPs), reduce side effects, realize controlled release of the contents, and enhanced targeted delivery [13]. SLNs are preferred as gene delivery systems due to their large surface areas, small dimensions, ability to transfect genetic material, biocompatibility, and suitability for large-scale production $[9,14]$. SLNs could be produced by various methods; high shear homogenization and ultrasound, high-pressure homogenization (hot and cold), solvent emulsification/evaporation, microemulsion based SLN preparations [15-19].

For the production of SLNs, the selection of components, their proportions, and the differences depending on the preparation method lead to the presence of a wide alternative sample space. Therefore, a method that allows a systematic analysis should be applied prior to experimental investigation. In a factorial design, the appropriate number of samples for various factors and levels are determined for the analysis of independent variables and dependent variables. The main goal is to achieve the highest level of output with the least number of data in order to reduce the labor force and cost while increasing efficiency. Box-Behnken designs are used to generate higher-order response surfaces using fewer required runs with factorial design technique. Box-Behnken design can also perform statistical analysis of the model, response optimization, and prediction of the formulations that meet the desired conditions $[20,21]$. In this study, we used a factorial design for scanning microemulsion components and optimizing SLN formulation. We examined the Design of Experiments (DOE), which we propose to be used in the development of formulations that are potential as gene delivery systems using Response Surface Methodology (RSM). Moreover, we characterized the complex formed by the optimal SLN formulation with plasmid DNA and examined it in terms of its protection potential against nucleases, cytotoxicity profile, and storage stability.

\section{Materials and Methods}

This study has been projected by the Scientific and Technological Research Council of Turkey 
(TÜBITTAK) under code TÜBİTAK-SBAG218S682. EGFP encoding plasmid pcDNA3EGFP was a gift from Doug Golenbock (Addgene plasmid \# 13031; http://n2t.net/addgene:13031; RRID: Addgene_13031). Behenoyl polyoxyl-8 glycérides (Compritol® HD5 ATO) (C-HD5) was kindly donated by Gattefosse, France. Macrogolglycerol ricinoleate (Kolliphor ${ }^{\circledR}$ ELP) (K-ELP) was kindly donated by BASF, Germany. Mouse fibroblast cell line (L929) was purchased from ATCC, USA. Ethanol (EtOH), Sodium dodecyl sulfate (SDS) were provided from Merck-Co., Germany. Dimethyldioctadecylammonium bromide (DDAB) was purchased from Sigma-Aldrich Co., USA. Alamar Blue cell viability assay kit was purchased from Thermo Fisher Scientific, USA. All other chemicals were of analytical grade and used as received. Ultrapure water was used in all stages needed.

\section{Nanoparticle preparation}

Cationic SLNs were produced with slight modifications to the previously described meltemulsification technique [22,23]. As formulation components, C-HD5 and DDAB were used as lipid phase; K-ELP was used surfactant (S); EtOH was used as co-surfactant $(\mathrm{CoS})$, and $\mathrm{UPH}_{2} \mathrm{O}$ was used as an aqueous phase. In the first step of production, microemulsion contents were weighed in a sealed cap glass vial as determined by factorial design based on preliminary laboratory studies. Then, the vial and its content were kept in a water bath at 10 degrees above the lipid melting point $\left(75^{\circ} \mathrm{C}\right)$ until transparent microemulsion was self-established. Obtained oil in water $(\mathrm{o} / \mathrm{w})$ microemulsion was dispersed in cold ultra-pure distilled water $\left(0-4{ }^{\circ} \mathrm{C}\right)$ at a ratio of $1: 10(\mathrm{w} / \mathrm{v})$ under vigorous stirring. SLNs were obtained by sudden freezing of microemulsion droplets in cold water.

\section{Experimental design}

Screening and optimization of SLN formulations were carried out with factorial design with 3 factors and 3 levels. The amount of lipid ( $3 \%, 4 \%$, and $5 \%)$ and the total amount of surfactant and co-surfactant $(18 \%, 24 \%$, and $30 \%)$ were selected as two variables. These variables are important since they form two main corners of the pseudo-ternary phase

Table 1. Variables and their levels in Box-Behnken Design.

\begin{tabular}{|l|l|l|l|l|}
\hline \multicolumn{2}{|l|}{ Variables } & \multicolumn{2}{l|}{ Level of variables } \\
\hline \multicolumn{2}{|l|}{ Independent variables } & Low Level (-1) & Mid-Level (0) & High Level (1) \\
\hline A & Lipid (\%) & $3 \%$ & $4 \%$ & $5 \%$ \\
\hline B & Surfactant + Co-Surfactant (1:2,w/w) (\%) & $18 \%$ & $24 \%$ & $30 \%$ \\
\hline C & Lipid to cationic lipid ratio (w/w) & $2: 1$ & $1: 1$ & $1: 2$ \\
\hline Dependent variables & Goals & & \\
\hline Y1 & Particle Size (nm) & Minimize & \\
\hline Y2 & Polydispersity Index & Minimize & \\
\hline Y3 & Zeta Potential (mV) & Target 35 mV & \\
\hline
\end{tabular}


Table 2. Designed formulation parameters and measured responses using Box-Behnken Desing for SLN production.

\begin{tabular}{|l|l|l|l|l|l|l|l|}
\hline No & Formulation Code & $\mathbf{A}$ & $\mathbf{B}$ & $\mathbf{C}$ & $\mathbf{Y 1}$ & Y2 & Y3 \\
\cline { 3 - 8 } & $\begin{array}{l}\text { Lipid } \\
\mathbf{( \% )}\end{array}$ & $\begin{array}{l}\text { S } \mathbf{( \mathbf { C } )} \\
\mathbf{( \% )}\end{array}$ & $\begin{array}{l}\text { L/Cat.L } \\
\mathbf{( w / w )}\end{array}$ & $\begin{array}{l}\text { Particle Size } \\
\mathbf{( n m )} \pm \text { S.D. }\end{array}$ & PDI \pm S.D. & $\begin{array}{l}\text { Zeta Potential } \\
\text { (mV) } \pm \text { S.D. }\end{array}$ \\
\hline $\mathbf{1}$ & C-HD5-SLN 1 & 3 & 24 & $2: 1$ & $3155 \pm 116$ & $0.756 \pm 0.060$ & $33.8 \pm 7.87$ \\
\hline $\mathbf{2}$ & C-HD5-SLN 2 & 3 & 24 & $1: 2$ & $200.4 \pm 29.37$ & $0.341 \pm 0.070$ & $34.6 \pm 2.67$ \\
\hline $\mathbf{3}$ & C-HD5-SLN 3 & 5 & 24 & $2: 1$ & $3513 \pm 117.1$ & $0.983 \pm 0.046$ & $64.6 \pm 4.09$ \\
\hline $\mathbf{4}$ & C-HD5-SLN 4 & 5 & 24 & $1: 2$ & $146.1 \pm 15.6$ & $0.239 \pm 0.042$ & $41.4 \pm 8.25$ \\
\hline $\mathbf{5}$ & C-HD5-SLN 5 & 4 & 18 & $2: 1$ & $4024 \pm 470.2$ & $0.858 \pm 0.202$ & $59.8 \pm 0.96$ \\
\hline $\mathbf{6}$ & C-HD5-SLN 6 & 4 & 18 & $1: 2$ & $65.31 \pm 2.578$ & $0.628 \pm 0.038$ & $40.0 \pm 3.12$ \\
\hline $\mathbf{7}$ & C-HD5-SLN 7 & 4 & 30 & $2: 1$ & $690.2 \pm 172.4$ & $0.740 \pm 0.150$ & $35.3 \pm 14.4$ \\
\hline $\mathbf{8}$ & C-HD5-SLN 8 & 4 & 30 & $1: 2$ & $45.86 \pm 16.63$ & $0.468 \pm 0.224$ & $40.6 \pm 3.96$ \\
\hline $\mathbf{9}$ & C-HD5-SLN 9 & 3 & 18 & $1: 1$ & $514.3 \pm 62.17$ & $0.531 \pm 0.082$ & $61.5 \pm 2.40$ \\
\hline $\mathbf{1 0}$ & C-HD5-SLN 10 & 5 & 18 & $1: 1$ & $708.4 \pm 118.2$ & $0.561 \pm 0.108$ & $60.8 \pm 1.68$ \\
\hline $\mathbf{1 1}$ & C-HD5-SLN 11 & 3 & 30 & $1: 1$ & $592.7 \pm 92.47$ & $0.596 \pm 0.076$ & $36.3 \pm 2,85$ \\
\hline $\mathbf{1 2}$ & C-HD5-SLN 12 & 5 & 30 & $1: 1$ & $222.1 \pm 11.95$ & $0.459 \pm 0.122$ & $39.9 \pm 3.13$ \\
\hline $\mathbf{1 3}$ & C-HD5-SLN 13 & 4 & 24 & $1: 1$ & $399.3 \pm 19.44$ & $0.516 \pm 0.291$ & $34.9 \pm 2.73$ \\
\hline $\mathbf{1 4}$ & C-HD5-SLN 14 & 4 & 24 & $1: 1$ & $353.1 \pm 62.41$ & $0.573 \pm 0.081$ & $33.5 \pm 3.48$ \\
\hline $\mathbf{1 5}$ & C-HD5-SLN 15 & 4 & 24 & $1: 1$ & $424.5 \pm 152.4$ & $0.573 \pm 0.006$ & $32.8 \pm 1.66$ \\
\hline
\end{tabular}

diagram, which is commonly used in the production of the microemulsion [24,25]. The presence and amount of cationic lipids, which are essential for cationic SLN production, were aimed to be investigated as a third variable. For this, the lipid to cationic lipid ratio $(2: 1,1: 1$, $1: 2, \mathrm{w} / \mathrm{w}$ ) was determined as the third variable. Dependent on the preliminary studies and according to the literature, the factorial design matrix was created using the Minitab 19 software (Minitab LLC, USA) using the variables represented in Table 1 . Totally 15 different compositions were determined as 12 alternative microemulsion contents and 3 midpoint replicates. Dynamic light scattering (DLS) analysis is widely used for the characterization of SLNs. Particle size, PDI, and zeta potential measurements obtained by DLS analysis were selected as dependent variables. Response optimization was determined to aim the smallest particle size and the most homogeneous particle size distribution in the manner of the lowest PDI value. Zeta potential was set as a target response value and determined as $35 \mathrm{mV}$. The designed variables with codes and actual responses in terms of DLS measurement results are given in Table 2. In order to provide screening in the sample space and according to the analysis of variance (ANOVA), the quadratic model was chosen for a Box-Behnken design. With the RSM analysis, P-Value, F-Value, coefficient determinant $\left(\mathrm{R}^{2}\right)$, 
and adjusted coefficient determinant ( $\mathrm{R}^{2}$ adj) data were evaluated statistically. Actual data of the formulation determined by response optimization were measured and the statistically produced predicted values were calculated. The fit of the model and secondorder polynomial equations were determined. Then, the 3D-surface and contour plots were analyzed.

Agarose gel retardation assay, SDS-induced release, and DNase I protection studies

Agarose gel retardation assay was performed to determine the optimal complex formation ratio of C-HD5-SLN and pcDNA3-EGFP. Complexes with various ratios were formed by mixing the constant amount of pcDNA3-EGFP $(100 \mathrm{ng} / \mu \mathrm{L})$ with the increasing amount of CHD5-SLN under gentle shaking for $30 \mathrm{~min}$ at room temperature, which allows the formation of electrostatic interactions between the positive charges of SLNs and the negative charges of pcDNA3-EGFP. The resultant complexes were characterized by agarose gel retardation assay [26-28].

In order to show the release of pcDNA3-EGFP, SDS induced release study was performed. To evaluate the capacity of the C-HD5-SLN to protect pcDNA3-EGFP, complexes were incubated with DNase I (0.4 IU DNase I/1 $\mu \mathrm{g}$ pDNA) at $37^{\circ} \mathrm{C}$ for 30 minutes, then decomplexed in presence of SDS $1 \%(\mathrm{w} / \mathrm{v})$ and further subjected agarose gel retardation assay [29]. The bands were observed by a UV transilluminator with a digital imaging system (Vilber Lourmat, France).

\section{Physicochemical characterization for freshly prepared SLNs and stability studies}

The particle size, polydispersity index, and zeta potential values of C-HD5-SLN and C-HD5SLN:pcDNA3-EGFP complex were measured by DLS (Zeta sizer Nano ZS, Malvern Instruments Ltd., UK) method using noninvasive backscattering mode with the detector positioned at $173^{\circ}$. DLS measurements were reported as averaged intensity weighted distribution for particle size measurements. The refractive index of the Compritol (1.456) as a main ingredient of the lipid matrix was used for DLS calculations [30]. The triplicate measurements were performed using disposable polystyrene microcuvettes.

The morphology of C-HD5-SLN and C-HD5SLN:pcDNA3-EGFP complex were visualized by using Scanning Electron Microscope (SEM, Carl Zeiss 300VP, Germany). Sample preparation was done by drying the nanoparticles on metal plates and then coating with $100 \mathrm{~A}^{\circ}$ thick gold in the brand coating device (Quorum Q150 Res, UK).

The physicochemical stability of the formulation was followed up to 2 months. SLNs were stored at $4{ }^{\circ} \mathrm{C}$ and measured at days 0,7 , 14,30 , and 60 to evaluate the stability.

\section{Cytotoxicity analysis}

The in vitro cytotoxicity profile of the C-HD5SLN and C-HD5-SLN:pcDNA3-EGFP complex was evaluated on the fibroblast cell line (L929). One day before the experiment, L929 cells were plated in 96-well plates at a density of $5 \times 10^{3}$ cells per well in $100 \mu \mathrm{L}$. After overnight incubation, cells were treated with $\mathrm{C}$ HD5-SLN and C-HD5-SLN:pcDNA3-EGFP complex at increasing formulation volumes (3, $6,9,12,15 \mu \mathrm{L} /$ well), with respect to SLN volume for $24 \mathrm{~h}$. The percentage of living cells was investigated by the Alamar Blue proliferation kit (Thermo Fisher Scientific, USA) according to the kit's manual.

\section{Statistical analysis}

Except for the experimental design and RSM, the data analysis was performed with Prism 6 
(GraphPad Software, Inc., USA) software. The statistical analysis between different groups has been assessed by a non-paired t-test and Twoway ANOVA followed by multiple comparison tests. Differences were considered statistically significant if the $P$ value was less than 0.05 . The results of all experiments were reported as mean \pm S.D.

\section{Results and Discussion}

Microemulsion content is the main factor determining the physicochemical properties of SLNs obtained by the microemulsion dilution method. With this method, the first step in the production of SLN is to obtain clear transparent microemulsions. One of the most common methods to obtain clear microemulsions is to is required to decide which point to choose. In order to make this statistical evaluation, dependent and independent variables and related responses should be determined. Particle size is the most widely used variable for SLN evaluation. While encapsulation efficiency is an eligible parameter for SLNs loaded with drugs, the zeta potential value is an important parameter for cationic SLNs that will interact with nucleic acids via electrostatic forces on the surface.

On the pseudo-ternary phase diagrams used in the conventional determination of the content ratios of the microemulsion, each corner has one of the main components of the formulation. In most cases, these are the lipid phase, the aqueous phase, and the total of surfactant and

Table 3. The fit of the model and second order polynomial equations of the responses

\begin{tabular}{|c|c|c|c|c|c|}
\hline \multirow[t]{2}{*}{ Response } & \multicolumn{4}{|c|}{ Regression analysis of variance } & \multirow[t]{2}{*}{ Regression equation in coded units } \\
\hline & $R^{2}$ & $R^{2} a d j$ & F-Value & P-Value & \\
\hline $\begin{array}{l}\text { Particle } \\
\text { Size }\end{array}$ & $94.19 \%$ & $83.73 \%$ & 376.83 & 0.003 & $\begin{array}{l}\mathrm{Y} 1=1824-4268 \mathrm{C}-2077 \mathrm{~A}+303 \mathrm{C} \\
+1029 \mathrm{C} * \mathrm{C}+332 \mathrm{~A} * \mathrm{~A}-5.98 \mathrm{~B} * \mathrm{~B} \\
-103 \mathrm{C}^{*} \mathrm{~A}+138.1 \mathrm{C} * \mathrm{~B}-23.5 \mathrm{~A} * \mathrm{~B}\end{array}$ \\
\hline PDI & $88.70 \%$ & $68.37 \%$ & 6.16 & 0.143 & 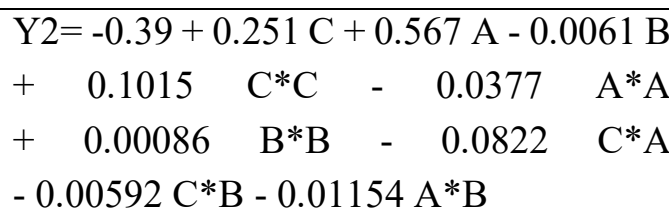 \\
\hline $\begin{array}{l}\text { Zeta } \\
\text { Potential }\end{array}$ & $88.62 \%$ & $78.14 \%$ & 64.12 & 0.015 & $\begin{array}{l}\mathrm{Y} 3=319.9-5.7 \mathrm{C}-61.5 \mathrm{~A}-12.99 \mathrm{~B} \\
+2.08 \mathrm{C}^{*} \mathrm{C}+7.78 \mathrm{~A}^{*} \mathrm{~A}+0.2252 \mathrm{~B} * \mathrm{~B} \\
-6.00 \mathrm{C}^{*} \mathrm{~A}+1.046 \mathrm{C}^{*} \mathrm{~B}+0.179 \mathrm{~A}^{* B}\end{array}$ \\
\hline
\end{tabular}

obtain pseudo-ternary phase diagrams and determine the microemulsion formation region on this diagram [24,25,31]. Then, SLNs are produced by selecting an initial microemulsion formulation based on the central point or several different points of this area. However, there can be an infinite number of alternative microemulsion formulation, regardless of the size of the area. A reliable statistical evaluation co-surfactant amounts in percentages. Among these factors, the lipid phase and total of surfactant and co-surfactant were selected as formulation independent variables (Table 1). The aqueous phase was not chosen as a variable because determining the percentage of lipid and the percentage of total surfactant and cosurfactant ensures that the percentage of water is also determined since the total of the system 
will be equal to one hundred percent. Therefore, the solid lipid/cationic lipid ratio in the lipid phase was chosen as the 3rd variable. The cationic lipid plays an important role in the charge of the formulation, hence its stability and its electrostatic interaction with negatively charged nucleic acids.

With this information, as indicated in Table 1, lipid percentage, total surfactant and cosurfactant percentage, and solid lipid cationic lipid ratio were determined as three independent variables. For the analysis of the response related to these variables, particle size, PDI, and zeta potential values were examined. Box-Behnken Design allows these variables to be evaluated with fewer samples. While it is necessary to test $3^{3}=27$ different microemulsions in 3-factor 3-level factorial design, with Box-Behnken Design optimization, this factorial design can be completed with only 15 experiments with 3 replicate center points. The contents of the microemulsion presented accordingly are given in Table 2 together with the response characterization values.

Response surface regression analysis was performed using the values in Table 2. The second order polynomial equations and statistical evaluations obtained accordingly are summarized in Table 3. According to the RSM analysis, the $\mathrm{F}$ value for the particle size was determined as $376.83(p=0.003<0.05)$. The $F$ value demonstrated that the selected quadratic model holds significance [20,21].

The coefficient determinant $\left(\mathrm{R}^{2}\right)$ value was found $94.19 \%$ value indicates the reliability of the model together with adjusted $\mathrm{R}^{2}$ value. Overall, it can be predicted that particle size is affected by all variables. The same interference is valid for zeta potential depending on the statistical data presented in Table 3. However, PDI value does not fit the model while F value was found to be 6.16 and the P-value is 0.145 $>0.05$.

When we investigate the surface and contour plots of response surface regression analysis in terms of particle size, PDI, and zeta potential, we observed that the surface and contour plots lines are curved. If the model showed no interaction, the 3D surface plots would be planar $[32,33]$. Curved structures are the sign of interaction between variables. Likewise, in contour plots, this situation can be demonstrated by the presence of elliptical lines. These inclined plots are indicative of a secondorder polynomial equation for the model (Figure 1,2,3). In the polynomial equation, the negative sign coefficient value indicates the antagonist effect and positive values indicate the agonist or synergist effect [20,34]. Regression Equation in Coded Units was given in Table 3. For particle size, $\mathrm{A}, \mathrm{C} * \mathrm{C}, \mathrm{A} * \mathrm{~A}$, and $\mathrm{C} * \mathrm{~B}$ values have positive coefficient values while $\mathrm{B}, \mathrm{C}, \mathrm{B} * \mathrm{~B}, \mathrm{C} * \mathrm{~A}$, and $\mathrm{A} * \mathrm{~B}$ values have negative coefficient values. In terms of $\mathrm{PDI}, \mathrm{A}, \mathrm{C}, \mathrm{C}^{*} \mathrm{C}$, and $\mathrm{B} * \mathrm{~B}$ values have positive coefficient values while $B, A^{*} A, C^{*} A$, $\mathrm{C} * \mathrm{~B}$, and $\mathrm{A} * \mathrm{~B}$ values have negative coefficient values. And for Zeta potential, $C^{*} \mathrm{C}, \mathrm{A}^{*} \mathrm{~A}, \mathrm{~B} * \mathrm{~B}$, $\mathrm{C}^{*} \mathrm{~B}$, and $\mathrm{A} * \mathrm{~B}$ values have positive coefficient values while $A, B, C$, and $C^{*} A$ values have negative coefficient values.

RSM helps in the prediction of the theoretical optimum conditions for desired responses [20,21,32]. Mathematically offered formulation components were experimentally obtained. Optimal desired formulation component ratios and, predicted and actual experimental values were shown in Table 4. The obtained data successfully predicts the responses in parallel significance with $P$ values. The particle size and zeta potential values of this formulation, coded as C-HD5-Opt-SLN, are very close to the predicted value. The PDI value was relatively 

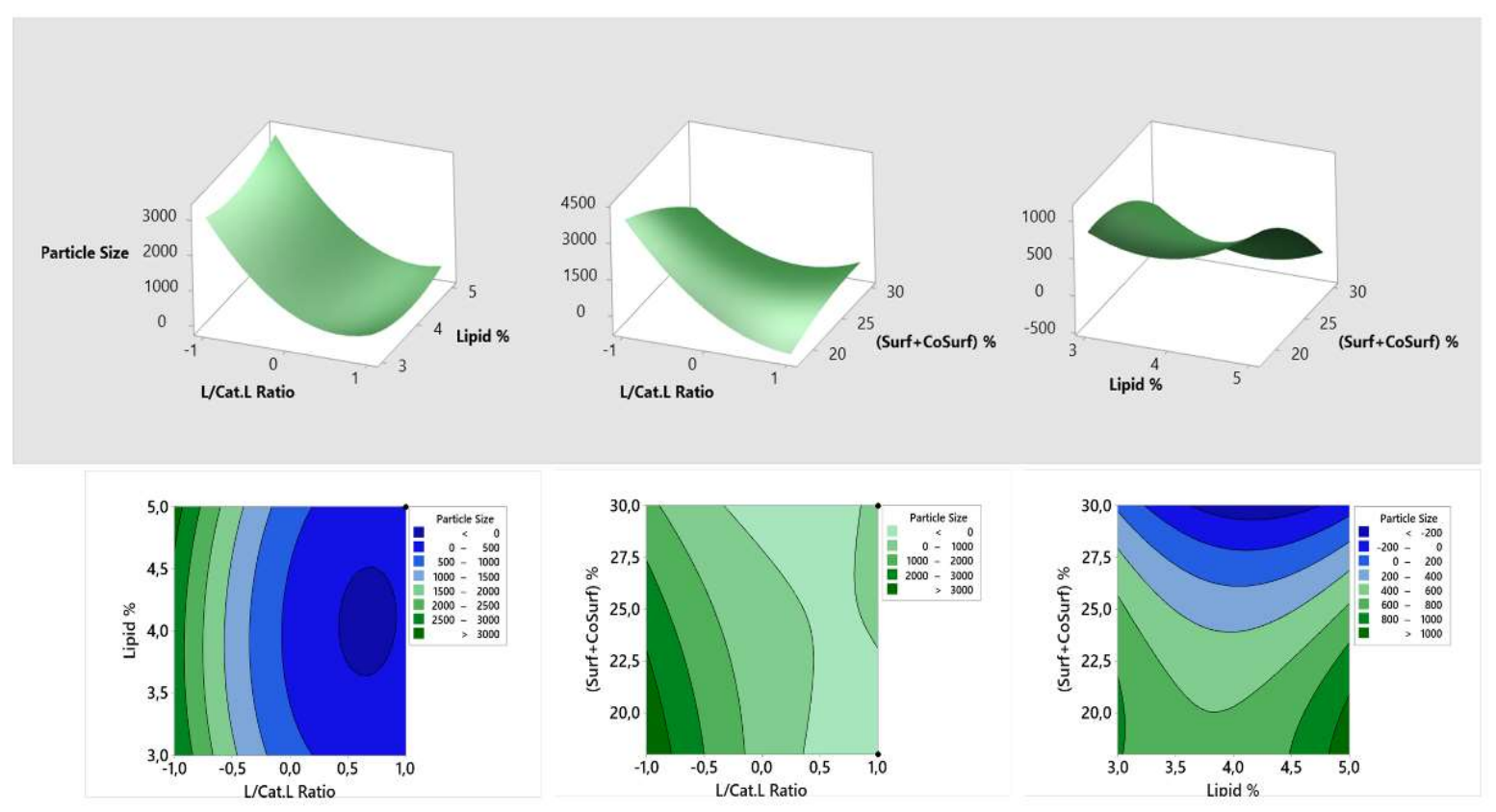

Figure 1. 3D surface response plots and contour plots showing the interference of the variables on particle size.

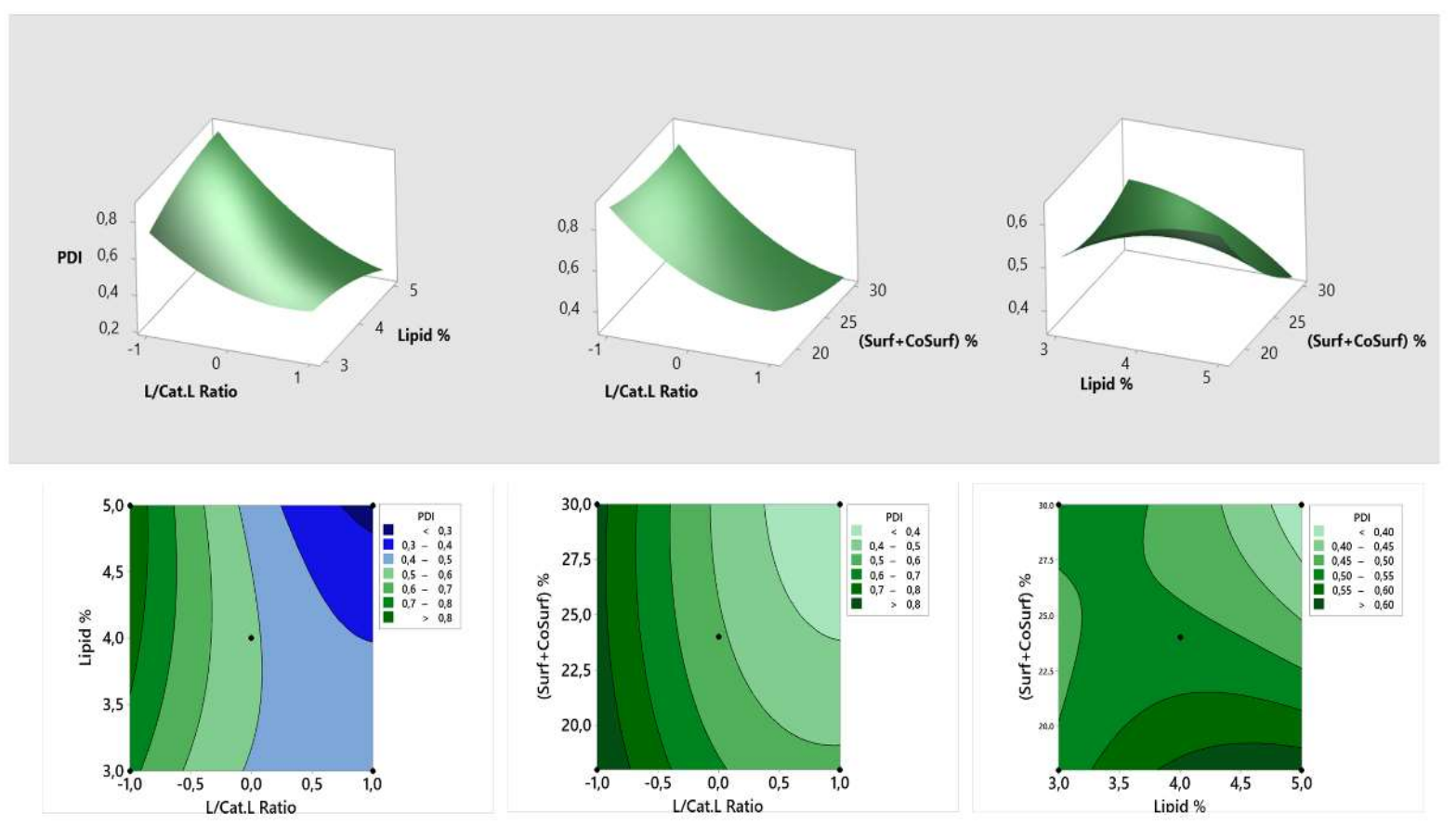

Figure 2. 3D surface response plots and contour plots showing the interference of the variables on PDI. 
far from the predicted value, as it was shown in the regression analysis that the PDI value was not fit for the model $(\mathrm{P}>0.05)$. Following the determination of consistency in optimized formulation values with the predicted values, the potential of this optimal SLN formulation was investigated in terms of the gene delivery system requirements.
RSM helps in the prediction of the theoretical optimum conditions for desired responses [20,21,32]. Mathematically offered formulation components were experimentally obtained. Optimal desired formulation component ratios and, predicted and actual experimental values were shown in Table 4. The obtained data successfully predicts the responses in parallel

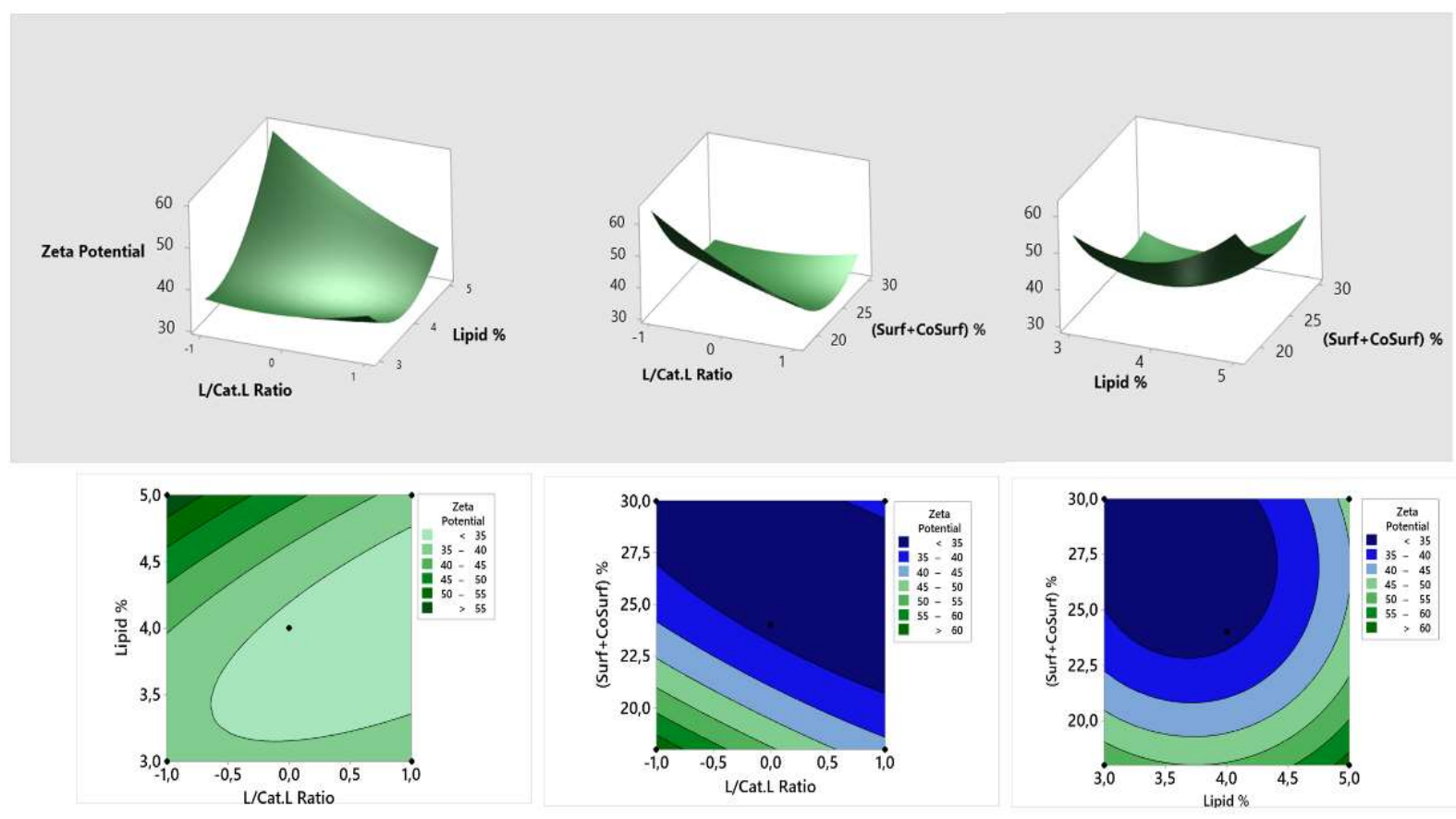

Figure 3. 3D surface response plots and contour plots showing the interference of the variables on zeta potential.

Table 4. Observed and predicted responses of the optimal formulation.

\begin{tabular}{|l|l|l|l|l|l|l|}
\hline \multirow{2}{*}{$\begin{array}{l}\text { C-HD5- } \\
\text { SLN-Opt }\end{array}$} & \multicolumn{3}{|l|}{ Optimized variables } & \multicolumn{3}{l|}{ Actual and predicted responses } \\
\cline { 2 - 7 } & $\mathrm{A}$ & $\mathrm{B}$ & $\mathrm{C}$ & $\mathrm{Y} 1$ & $\mathrm{Y} 2$ & $\mathrm{Y} 3$ \\
\hline $\begin{array}{l}\text { Predicted } \\
\text { Values }\end{array}$ & $\% 4.45$ & $\% 28.3$ & $\begin{array}{l}0.88(1: 1.83 \\
\mathrm{w} / \mathrm{w})\end{array}$ & 48.13 & 0.279 & 35.01 \\
\cline { 1 - 4 } $\begin{array}{l}\text { Actual } \\
\text { Values }\end{array}$ & & & & $51.37 \pm 0,2843$ & $0.209 \pm 0.005$ & $35.1 \pm 5.09$ \\
\hline
\end{tabular}


significance with $\mathrm{P}$ values. The particle size and zeta potential values of this formulation, coded as C-HD5-Opt-SLN, are very close to the predicted value. The PDI value was relatively far from the predicted value, as it was shown in the regression analysis that the PDI value was not fit for the model $(\mathrm{P}>0.05)$. Following the determination of consistency in optimized completely stopped at the ratio of $1: 1(\mathrm{v} / \mathrm{v})$ as seen in the fourth well. (Figure $4 \mathrm{a}$ ). The same ratios investigated for the SDS-induced release study and at all ratios, SLNs were released the pcDNA3-EGFP for the total concentration of $1 \%$ SDS (w/v) solution (Figure $4 b)$. In Figure $4 \mathrm{c}$, all complex ratios performed in electrophoretic mobility assay were prepared
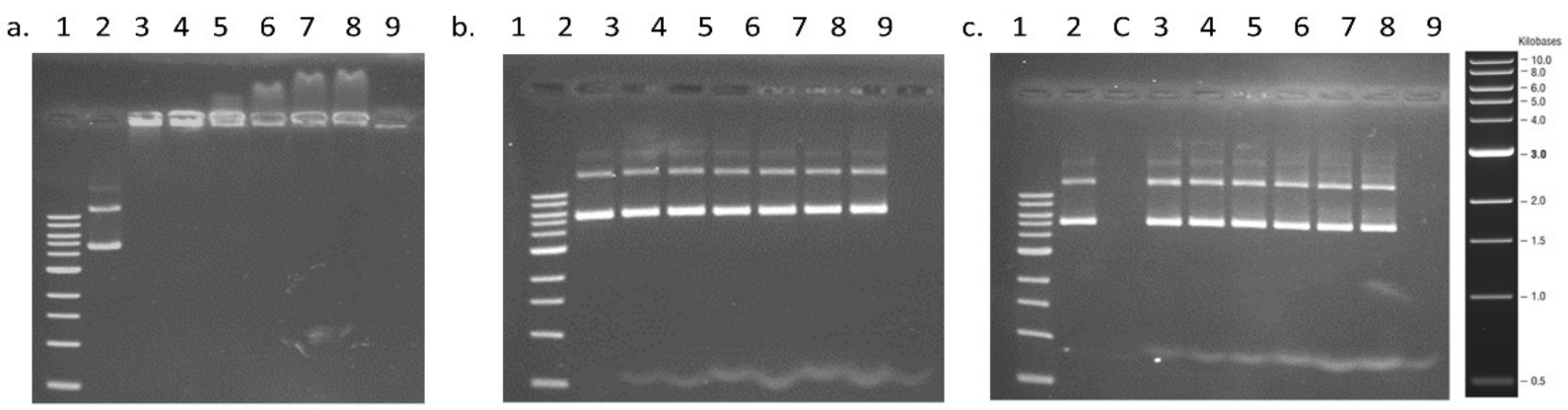

Figure 4 (a-c). Gel retardation, SDS-induced release and DNase I protection study agarose gel images. a) Complex formation ratios of C-HD5-SLN-Opt: pcDNA3-EGFP, b) SDS-induced release of C-HD5-SLN-Opt: pcDNA3-EGFP complexes, c) Enzymatic degradation of C-HD5-SLN-Opt: pcDNA3-EGFP. Lanes from left 1:1kb DNA ladder as molecular weight marker, 2: naked plasmid DNA (100ng/well), 3-7: C-HD5-SLN-Opt: pcDNA3-EGFP complexes (respectively 0.2:1, 0,5:1, 1:1, 2:1, 3:1, v/v). C: Naked pDNA degraded with DNase I enzyme as a control.

formulation values with the predicted values, the potential of this optimal SLN formulation was investigated in terms of the gene delivery system requirements.

Cationic SLNs developed as gene delivery systems form complexes with plasmid DNA via electrostatic interactions. However, the ratio of this complex should be determined to find optimal C-HD5-SLN-Opt: pcDNA3-EGFP ratio. For this, electrophoretic mobility assays were performed for the pcDNA3-EGFP plasmid. Samples were prepared by forming complexes with increasing amounts of C-HD5SLN-Opt, against a fixed amount of pcDNA3EGFP (100 ng) with vigorous shaking at room temperature. When the agarose gel electrophoresis images were examined, it was observed that the electrophoretic mobility freshly and then treated with DNase I (0.4 IU DNase I/1 $\mu \mathrm{g}$ pDNA) enzyme to determine the protection capacity of the formulation $[27,35]$. After incubation at $37^{\circ} \mathrm{C}$ for 30 minutes, SDSinduced release of pcDNA3-EGFP was performed on both samples and further subjected by agarose gel electrophoresis to compare the condition of the released plasmid. Naked pcDNA3-EGFP was treated with the same amount of DNase I as control of enzyme activity (Lane C). As shown in Figure 2c, the best protection was provided in starting 1:1 $(\mathrm{v} / \mathrm{v})$ ratio, which is seen in lane 4 and determined as optimal according to the electrophoretic mobility assay.

Next, the physicochemical properties of the complex (C-HD5-SLN-Opt: pcDNA3-EGFP, $1: 1, \mathrm{v} / \mathrm{v})$ were characterized. Particle size was 
again found nanosized and below $80 \mathrm{~nm}$. This size range is adequate for traverse into the cells [36]. Moreover, the charge of the system remained cationic. This is a factor that increases the entry of particles and genetic material into the cell by electrostatic interaction with the negatively charged cell membrane (Table 5). One of the precursors of an optimal and compact complex formation ratio is the PDI value. The PDI values below 0.4 indicate that the nanoparticle system is considered as monodispersed for drug delivery and PDI values over 0.7 indicate that the nanoparticle system has a broad size distribution for being a drug delivery system [23,37]. Another important parameter for the characterization of nanoparticles is the particle size distribution plot. For both C-HD5-SLN-Opt and C-HD5SLN-Opt: pcDNA3-EGFP $(1: 1, \mathrm{v} / \mathrm{v})$ complex, a single peak was observed (Figure 5). If the structure of the complex was not adequately compact, more than one peaks would have been observed representing the excessive amount of pcDNA3-EGFP or bare C-HD5-SLN-Opt.

Increasing the amount of C-HD5-SLN-Opt and C-HD5-SLN-Opt: pcDNA3-EGFP $(1: 1, \mathrm{v} / \mathrm{v})$ complex was evaluated for cytotoxicity test (Figure 6). In cytotoxicity studies, C-HD5SLN-Opt and C-HD5-SLN-Opt: pcDNA3EGFP $(1: 1, \quad v / v)$ complex containing an equivalent amount of nanoparticle were tested in L929 cells. Cytotoxicity profiles are parallel to each other for the nanoparticle and the complex. Although there is dose-dependent cytotoxicity in both cell lines, the cell viability for the required amounts for transfection studies has not fallen below $70 \%$. Delgado et al. used $2.5 \mu \mathrm{g} / \mathrm{mL}$ plasmid DNA for transfection. This corresponds to the use of the C-HD5-SLNOpt: pcDNA3-EGFP complex of less than $3 \mu \mathrm{L}$ sample volume used in the cytotoxicity study [38].

Table 5. Physicochemical characterization of optimal complex.

\begin{tabular}{|l|l|l|l|}
\hline Characterization of Complex & $\begin{array}{l}\text { Particle Size } \\
(\mathrm{nm}) \pm \text { S.D. }\end{array}$ & $\begin{array}{l}\text { PDI } \\
\pm \text { S.D. }\end{array}$ & $\begin{array}{l}\text { Zeta Potential } \\
(\mathrm{mV}) \pm \text { S.D. }\end{array}$ \\
\hline C-HD5-SLN-Opt: pcDNA3-EGFP (1:1, v:v) & $73.76 \pm 0.093$ & $0.253 \pm 0.007$ & $30.1 \pm 1.48$ \\
\hline
\end{tabular}

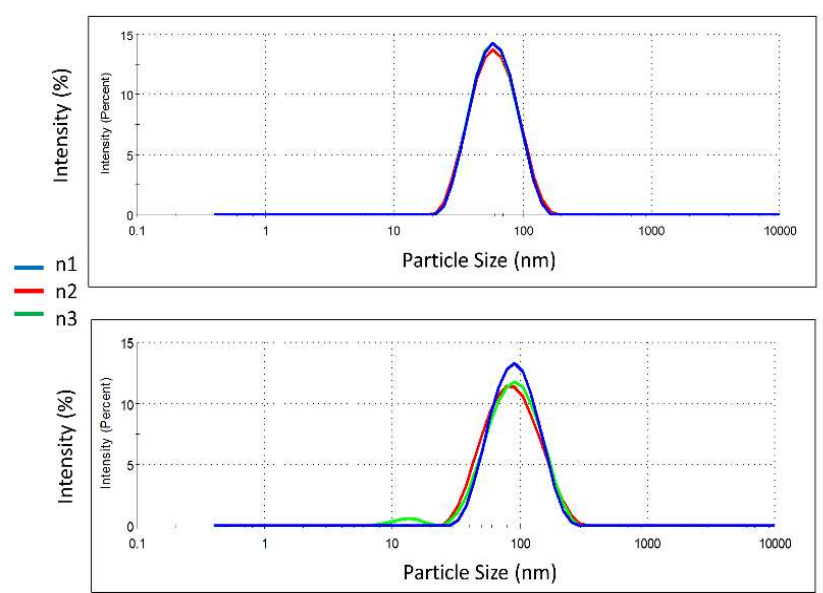

Figure 5. Size distribution plot of C-HD5-SLN-Opt and C-HD5-SLN-Opt: pcDNA3-EGFP, (1:1, $\mathrm{v} / \mathrm{v}$ ) complex. $\mathrm{n}=3$. 


\section{Cytotoxicity in L929 cells}

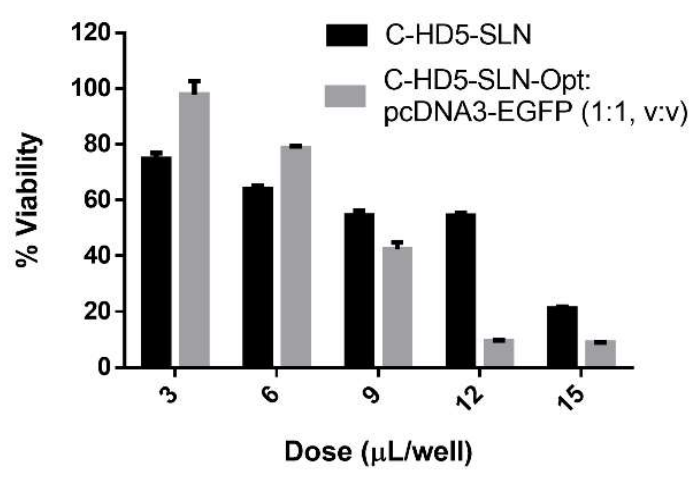

Figure 6. Viability percentages of L929 cells treated with increasing doses of C-HD5-SLNOpt and equivalent SLN doses of C-HD5-

SLN-Opt: pcDNA3-EGFP, $(1: 1, \mathrm{v} / \mathrm{v})$ complex.

The physicochemical stability of the developed C-HD5-SLN-Opt vector system was also evaluated. For this purpose, C-HD5-SLN-Opt were stored at $4{ }^{\circ} \mathrm{C}$ and particle size was measured at days $0,7,14,30$, and 60 (Figure 7). No significant increase was observed in particle size until day $21(\mathrm{p}>0.05)$. PDI value did not change significantly during the duration of stability monitoring.

\section{Stability of C-HD5-SLN-Opt}

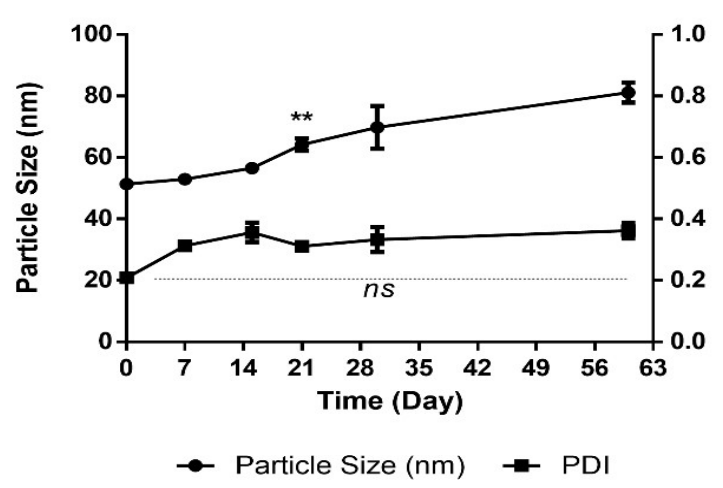

Figure 7. The storage stability of the developed C-HD5-SLN-Opt delivery system over 60 days at $4{ }^{\circ} \mathrm{C}$. Left $\mathrm{Y}$ axis represents the particle size and right $\mathrm{Y}$ axis represents the PDI of C-HD5-SLNOpt. ** represent $\mathrm{P}<0.01$, ns: not significant, $\mathrm{n}=3$.
Representative SEM photographs shows that of C-HD5-SLN-Opt and C-HD5-SLN-Opt: pcDNA3-EGFP, $(1: 1, \mathrm{v} / \mathrm{v})$ complex are in the global structure and their size is correlated with the DLS measurement (Figure 8). For the advanced characterization of C-HD5-SLN-Opt and C-HD5-SLN-Opt: pcDNA3-EGFP, (1:1, $\mathrm{v} / \mathrm{v})$ complex statistical evaluations can be enriched with various methods such as differential scanning calorimetry (DSC) analysis, permeability studies, and pharmacokinetic studies [19].
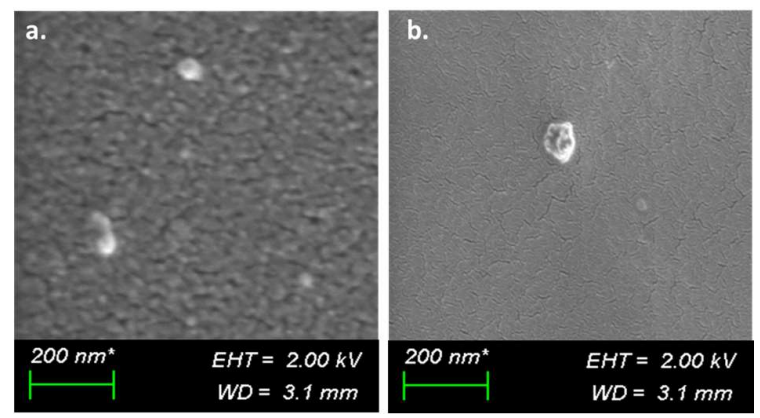

Figure 8. SEM images of (a) C-HD5-SLN-Opt and (b) C-HD5-SLN-Opt: pcDNA3-EGFP, $(1: 1, \mathrm{v} / \mathrm{v})$ complex.

\section{Conclusion}

The cationic solid lipid nanoparticles were successfully prepared using factorial design. For this purpose, Box Behnken design was used to optimize and analyze the formulation parameters. Response optimization was performed via software to find out the optimum conditions for desired SLN formulation. Optimum solid lipid nanoparticle formulation was investigated in terms of its protection potential against nucleases, cytotoxicity profile, and storage stability. In this regard, the obtained C-HD5-SLN-Opt coded solid lipid nanoparticle formulation provides a good basis as a gene delivery system. We showed that Box-Behnken design is a useful model to reduce labor force and cost especially for compounds with low quantities and high prices. With this method, it 
is possible to produce solid lipid nanoparticles with the desired feature by differing the variables and responses. Moreover, transfection efficiency can also be analyzed and incorporated into the Box-Behnken Design as a dependent variable and its efficiency can be optimized in the further studies.

\section{Acknowledgment}

This study has been financially supported by the Scientific and Technological Research Council of Turkey (TÜBITAK) under grant code TÜBITTAK-SBAG-218S682.

Conflict of Interest: None of the authors of this paper has a financial or personal relationship with other people or organizations that could inappropriately influence or bias the content of the paper.

No Competing interests are at stake and there is No Conflict of Interest" with other people or organizations that could inappropriately influence or bias the content of the paper.

\section{ORCID iD of the author(s)}

Hasan Akbaba / 0000-0001-9273-6346

Melike Ozder / 0000-0001-6150-7444

\section{Copyrights: (C) 2021@author (s).}

This is an open access article distributed under the terms of the Creative Commons Attribution License (CC BY 4.0), which permits unrestricted use, distribution, and reproduction in any medium, provided the original author(s) and source are credited and that the original publication in this journal is cited, in accordance with accepted academic practice. No use, distribution or reproduction is permitted which does not comply with these terms.

\section{References}

[1]Seoane-Vazquez E, Shukla V, RodriguezMonguio R. Innovation and competition in advanced therapy medicinal products. EMBO Mol Med. 2019;11(3):1-5.

[2]Ahmadi S, Rabiee N, Bagherzadeh M, et al. Stimulus-responsive sequential release systems for drug and gene delivery. Nano Today. 2020;34:100914.

[3]Kogure K, Akita H, Yamada Y, et al. Multifunctional envelope-type nano device (MEND) as a non-viral gene delivery system. Adv Drug Deliv Rev. 2008;60(45):559-571.

[4]Zhao J, Lu D, Moya S, et al. Bispecific Tcell engager (BiTE) immunotherapy of ovarian cancer based on MIL-88A MOF/MC gene delivery system. Appl Mater Today. 2020;20:100701.

[5]Büyükköröglu G, Yazan EY, Öner AF. Preparation and physicochemical characterizations of solid lipid nanoparticles containing DOTAPR $®$ for DNA delivery. Turk J Chem. 2015;39(5):1012-1024.

[6]Gascón AR, del Pozo-Rodríguez A, Solinís MA. In: Molina FM, editor. Non-Viral Delivery Systems in Gene Therapy, Gene Therapy - Tools and Potential Applications. London: IntechOpen; 2013. p. 1-33.

[7]Cevher E, Sezer AD, Çağlar ES. Gene Delivery Systems: Recent Progress in Viral and Non-Viral Therapy. In:Sezer AD, editor. Recent Advances in Novel Drug Carrier Systems. London: InTechOpen; 2012. p. 438-467.

[8]Rençber S, Köse FA, Karavana SY. Dexamethasone loaded PLGA nanoparticles for potential local treatment of oral precancerous lesions. Pharm Dev Technol. 2020;25(2):149-58.

[9]Limeres MJ, Suñé-Pou M, Prieto-Sánchez S, et al. Development and characterization of 
an improved formulation of cholesteryl oleate-loaded cationic solid-lipid nanoparticles as an efficient non-viral gene delivery system. Colloids Surf. B. 2019;184:110533.

[10]Labatut AE, Mattheolabakis G. Non-viral based miR delivery and recent developments. Eur J Pharm Biopharm. 2018;128:82-90.

[11] Şenyiğit ZA, Karavana SY, Eraç B, et al. Evaluation of chitosan based vaginal bioadhesive gel formulations for antifungal drugs. Acta Pharm. 2014;64(2):139-56.

[12] Das T, Venkatesh MP, Pramod-Kumar TM, et al. SLN based lendronate in situ gel as an implantable drug delivery system - A full factorial design approach. J Drug Deliv Sci Technol. 2020;55:101415.

[13]Zhang M, Guo X, Wang M, et al. Tumor microenvironment-induced structure changing drug/gene delivery system for overcoming delivery-associated challenges. J Control Release. 2020;323:203-224.

[14] Apaolaza PS, del Pozo-Rodríguez A, Solinís MA, et al. Structural recovery of the retina in a retinoschisin-deficient mouse after gene replacement therapy by solid lipid nanoparticles. Biomaterials. 2016;90:40-49.

[15]Mehnert W, Mäder K. Solid lipid nanoparticles: Production, characterization and applications. Adv Drug Deliv Rev. 2001;47(2-3):165-96.

[16]Hou DZ, Xie CS, Huang KJ, et al. The production and characteristics of solid lipid nanoparticles (SLNs). Biomaterials. 2003;24(10):1781-85.

[17] Üstündağ-Okur N, Çağlar EŞ, Arpa MD, et al. Preparation and evaluation of novel microemulsion-based hydrogels for dermal delivery of benzocaine. Pharm Dev Technol. 2017;22(4):500-10.
[18] Mehrabi M, Esmaeilpour P, Akbarzadeh A, et al. Efficacy of pegylated liposomal etoposide nanoparticles on breast cancer cell lines. Turk J Med Sci. 2016;46(2):567-71.

[19] Üstündağ-Okur N, Yurdasiper A, Gündoğdu E, et al. Modification of solid lipid nanoparticles loaded with nebivolol hydrochloride for improvement of oral bioavailability in treatment of hypertension: Polyethylene glycol versus chitosan oligosaccharide lactate. J Microencapsul. 2016;33(1):30-42.

[20]Kumar S, Narayan R, Ahammed V, et al. Development of ritonavir solid lipid nanoparticles by Box Behnken design for intestinal lymphatic targeting. J Drug Deliv Sci Technol. 2018;44 (September 2017):181-89

[21] Yasir M, Sara UVS. Preparation and optimization of haloperidol loaded solid lipid nanoparticles by Box-Behnken design. J Pharm Res. 2013;7(6):551-58.

[22] Das S, Chaudhury A. Recent advances in lipid nanoparticle formulations with solid matrix for oral drug delivery. AAPS PharmSciTech. 2011;12(1):62-76.

[23] Akbaba H, Erel-Akbaba G, Kantarc1 AG. Development and evaluation of antisense shRNA-encoding plasmid loaded solid lipid nanoparticles against 5- $\alpha$ reductase activity. J Drug Deliv Sci Technol. 2018;44 (August 2017):270-77.

[24]Fadda P, Monduzzi M, Caboi F, et al. Solid lipid nanoparticle preparation by a warm microemulsion based process: Influence of microemulsion microstructure. Int J Pharm. 2013;446(1-2):166-75.

[25] Erel-Akbaba G, Carvalho LA, Tian T, et al. Radiation-Induced Targeted NanoparticleBased Gene Delivery for Brain Tumor Therapy. ACS Nano. 2019;13(4):4028-40 
[26]Bi YZ, Zhang YF, Cui CY, et al. Genesilencing effects of anti-survivin siRNA delivered by RGDV-functionalized nanodiamond carrier in the breast carcinoma cell line MCF-7. Int $\mathrm{J}$ Nanomedicine. 2016;11:5771-5787.

[27] Vighi E, Ruozi B, Montanari M, et al. pDNA condensation capacity and in vitro gene delivery properties of cationic solid lipid nanoparticles. Int J Pharm. 2010;389(12):254-61.

[28]Alan S, Şalva E, Yılmaz İ, et al. The effectiveness of chitosan-mediated silencing of PDGF-B and PDGFR- $\beta$ in the mesangial proliferative glomerulonephritis therapy. Exp Mol Pathol. 2019;110:104280.

[29] del Pozo-Rodríguez A, Pujals S, Delgado D, et al. A proline-rich peptide improves cell transfection of solid lipid nanoparticle-based non-viral vectors. J Control Release. 2009;133(1):52-59.

[30] Souto EB, Mehnert W, Müller RH. Polymorphic behaviour of Compritol ${ }^{\circledR} 888$ ATO as bulk lipid and as SLN and NLC. J Microencapsul. 2006;23(4):417-33.

[31] Akbaba H, Kantarc1 AG, Erel-Akbaba G. Development and in vitro evaluation of positive-charged solid lipid nanoparticles as nucleic acid delivery system in glioblastoma treatment. Marmara Pharm J. 2018;22(2):299-306.

[32] Delan WK, Zakaria M, Elsaadany B, et al. Formulation of simvastatin chitosan nanoparticles for controlled delivery in bone regeneration: Optimization using BoxBehnken design, stability and in vivo study. Int J Pharm. 2020;577 (September 2019):119038.

[33] Aslam M, Aqil M, Ahad A, et al. Application of Box-Behnken design for preparation of glibenclamide loaded lipid based nanoparticles: Optimization, in vitro skin permeation, drug release and in vivo pharmacokinetic study. J Mol Liq. 2016;219:897-908.

[34] Wang L, Wang CY, Zhang Y, et al. Preparation and characterization of solid lipid nanoparticles loaded with salmon calcitonin phospholipid complex. J Drug Deliv Sci Technol. 2019;52:838-45.

[35]del Pozo-Rodríguez A, Delgado D, Solinís MÁ, et al. Solid lipid nanoparticles as potential tools for gene therapy: In vivo protein expression after intravenous administration. Int J Pharm. 2010;385(12):157-62.

[36] Salva E, Turan SÖ, Akbuğa J. Inhibition of Glomerular Mesangial Cell Proliferation by siPDGF-B- and siPDGFR- $\beta$-Containing Chitosan Nanoplexes. AAPS PharmSciTech. 2017;18(4):1031-42.

[37]Danaei M, Dehghankhold M, Ataei S, et al. Impact of particle size and polydispersity index on the clinical applications of lipidic nanocarrier systems. Pharmaceutics. 2018;10(2):1-17.

[38]Delgado D, Gascón AR, del Pozo-Rodríguez $A$, et al. Dextran-protamine-solid lipid nanoparticles as a non-viral vector for gene therapy: In vitro characterization and in vivo transfection after intravenous administration to mice. Int J Pharm. 2012;425(1-2):35-43. 